\title{
Molecular cloning and characterization of GbMECT and GbMECP gene promoters from Ginkgo biloba
}

\author{
S.Y. Cheng ${ }^{1 *}$, L.L. Li ${ }^{2 *}$, H.H. Yuan ${ }^{1,2}$, F. Xu ${ }^{3}$ and H. Cheng ${ }^{1,2}$ \\ ${ }^{1}$ School of Biology and Pharmaceutical Engineering, Wuhan Polytechnic University, \\ Wuhan, Hubei, China \\ ${ }^{2}$ Economic Forest Germplasm Improvement and Comprehensive Utilization of \\ Resources of Hubei Key Laboratories, Hubei, Huanggang, China \\ ${ }^{3}$ College of Horticulture and gardening, Yangtze University, Jingzhou, Hubei, China \\ ${ }^{*}$ These authors contributed equally to this study. \\ Corresponding author: H. Cheng \\ E-mail: chenghua1437@126.com
}

Genet. Mol. Res. 14 (4): 15112-15122 (2015)

Received June 22, 2015

Accepted October 13, 2015

Published November 24, 2015

DOI http://dx.doi.org/10.4238/2015.November.24.20

ABSTRACT. Ginkgolides are key pharmaceutical components in Ginkgo biloba. Using the cDNA sequence of the MECP and MECT genes to design primers, we obtained the promoters of these genes from Ginkgo genomic DNA using the genome walking method. The two promoters were 744 and $982 \mathrm{bp}$ in length, respectively. The cis-elements of the GbMECPs and GBMECT promoters were predicted and analyzed using the plant cis-acting regulatory element database. We found major cis-elements in the sequence of the GbMECT and GbMECPs promoters. The GbMECP promoter contains six TATA boxes and eight CAAT boxes. The GbMECT contains five TATA boxes and seven CAAT boxes. Furthermore, some ciselements in the promoters of GbMECPs and GbMECT included hormone and light-regulated elements, UB-B-induced elements, and stress-related dehydration-responsive elements. Expression analysis results showed that the MECP gene is mainly involved in responses to CCC (cycocel) and UV- 
$B$, and that MECT is mainly involved in responses to wounding treatment. These results also showed that the expression model was consistent with the cis-elements present. During the annual growth cycle, the level of GbMECPs was significantly correlated with terpene lactones accumulation in leaves. A fitted quadratic curve showed the best model for correlating GbMECPs with terpene lactones in leaves. These results will help us to understand the transcriptional regulatory mechanisms involved in key gene expression and ginkgolide accumulation in G. biloba.

Key words: Cis-acting elements; Hormone; Wounding; Diterpenoids; Terpene lactones; Functional analysis

\section{INTRODUCTION}

In recent years, Ginkgo biloba has become one of the most popular functional plants, especially for medicinal purposes. Extracts of G. biloba leaves contain active compounds such as flavonoids and terpene lactones (especially ginkgolides and bilobalide), and can, therefore, be used to increase peripheral and cerebral blood flow (Smith and Luo, 2004). Terpenoids are the most structurally diverse group of plant natural products (Kang et al., 2013). Although ginkgolides are considered to play a key role in the active ingredients of ginkgo, the concentration of ginkgolides is very low, at $0.25 \%$ in leaves (van Beek and Montoro, 2009). Therefore, terpene trilactone content should be increased in order to improve the medicinal and commercial value of ginkgo leaf production.

Plants use both the classical mevalonic acid (MVA) pathway and the 2-C-methyl-Derythritol 4-phosphate (MEP) pathway for isoprenoid biosynthesis, although they are localized in different compartments (Lichtenthaler, 1999). Anabolism of ginkgo terpene trilactones occurs mainly through the MEP pathway. The enzyme involved in the fifth step of the MEP pathway is 2-C-methylerythritol 2,4-cyclodiphosphate synthase, which is encoded by the Mecps gene (Bick and Lange, 2003). Experimental data have revealed that MECPs participates in the control of isoprenoid accumulation in plants, A correlation between MECPs expression and terpenoid accumulation has also been demonstrated (Veau et al., 2000). 2-C-methyl-D- erythritol 4phosphate Cytidyltransferase (MECT) is the third enzyme in the MEP pathway, which catalyzes the formation of 4-(cytidine 5'-diphospho)-2-C-methyl-D-erythritol from MEP and has been cloned and characterized from G. biloba embryonic roots (Kim et al., 2006). The expression of MECP and MECT genes is elaborately regulated by its growth as well as internal and external factors, as evidenced by the complex interaction between transcription factors and acting elements. Interaction between the expression of regulatory elements upstream of the two genes and trans-acting factors clearly merits further investigation as it will help regulate the accumulation of Ginkgo terpene. In this study, the upstream transcription regulatory sequence of the two genes, GbMECP and GbMECTs, were cloned via genome walking technology and bioinformatics analysis was performed. The two genes expression model were detected under different stress treatments, and also evaluated the relationship with terpene lactone in annual growth year in Ginkgo leaves. Studies investigating the role of the regulatory sequences in structural gene expression during synthetic metabolism in ginkgolides are crucial for identifying the molecular mechanisms involved in this process. 


\section{MATERIAL AND METHODS}

\section{Plant material and treatments}

Twelve-year-old grafted G. biloba seedlings were growing in a greenhouse in Huanggang (E, $114^{\circ} 54^{\prime}-116^{\circ} 8^{\prime}, \mathrm{N}, 2^{\circ} 45^{\prime}-31^{\circ} 35^{\prime}$, Hubei province, east of China) were sampled and used to isolate GbMECPs and MECT promoters. Seedlings from the same genotypic strain of G. biloba were subjected to treatments with UV-B, wounding (WOU), and chlormequat (CCC). For UV-B treatment, seedlings were exposed to $1500 \mathrm{~J} / \mathrm{m}^{2}$ UV-B irradiation in a closed chamber, and the control cuttings were placed in a dark closed chamber. The edges of leaves were wounded by cutting $\sim 0.5 \mathrm{~cm}$ with scissors, the intact leaves of seedling were used as the control. CCC (10 $\mathrm{mM}$ ) was dissolved in $0.01 \%$ Tween 20 and sprayed onto cutting leaves. The control leaves were sprayed with an equivalent volume of $0.01 \%(\mathrm{v} / \mathrm{v})$ Tween 20 . All samples were immediately frozen in liquid nitrogen and stored at $-80^{\circ} \mathrm{C}$ prior to RNA extraction and gene expression analysis. The different oligonucleotides used in this work are described in Table 1.

Table 1. Genomewalker and expression analysis primers.

\begin{tabular}{|c|c|c|}
\hline Description & Serial number & Sequences $\left(5^{\prime}-3^{\prime}\right)$ \\
\hline \multirow[t]{2}{*}{ Adaptor primers } & AP1 & GTAATACGACTCACTATAGGGC \\
\hline & AP2 & ACTATAGGGCACGCGTGGT \\
\hline \multirow[t]{8}{*}{ Primers for promoter isolation } & MT1 & GTGAGGAGACAATGAACTGGGAAC \\
\hline & MT2 & CTGGGAACGGTGTTACATGCTA \\
\hline & MT3 & CATCGTCATCATCTTCATAGACTTCGGC \\
\hline & MT4 & TAAGGACAATAGGAGGAAAAGTA \\
\hline & MPS1 & CTGATGA TTATGAACCCAACTGATT \\
\hline & MPS2 & CAACTGATTGCAGATTTGAGAGAT \\
\hline & MPS3 & GGTC TCGCT TATGCGGTTCCATGTTCC \\
\hline & MPS4 & TAGTTAGGTATCAAGGTCTCCGT \\
\hline \multirow[t]{6}{*}{ Primers for verification } & MECT1 & AATGCTTGTAGACTCTTCTTCTACTTTCC \\
\hline & MECT2 & ATGTCATGTCATTTGAGACTTACG \\
\hline & MPC & GATCGATCTGGAACGATTGATGGAATCCGA \\
\hline & MPB & AGGATCCACGCTCTTGCGGTCGTACTATGC \\
\hline & MPU & CGAGTGCCAACGACCATGA AT \\
\hline & MPD & ACCCACACTTTGCCGTAATGAG \\
\hline \multirow[t]{6}{*}{ Primer for RQPCR } & MET1 & TAAGGGTGCTGATTCTGGGACG \\
\hline & MET2 & TTCСTCСTGCCAATAGAACAAC \\
\hline & MEP1 & CAGTTTTCTTGAAGGAGGCGGT \\
\hline & MEP2 & TAGCCTCTTTATGGGGACTCAA \\
\hline & GAPU & TAGGAATCCCGAGGAAATACC \\
\hline & GAPD & TTCACGCCAACAACGAACATG \\
\hline
\end{tabular}

\section{Relative quantification by $\mathrm{qRT}$-PCR}

The levels of GbMECT and GbMECP transcripts were determined in different G. biloba tissues, as well as in leaf samples from young seedlings collected at different time points after stress and hormone treatment. qRT-PCR was carried out using an ABI PRISM 7500 Sequence Detection System (Applied Biosystems, USA) with SYBR Green PCR Master Mix (Applied Biosystems, USA) according to the manufacturer protocol. GbMECT and GbMECP sequences were obtained from NCBI database supplyed by Kim(2006) and Gao(2006). The G. biloba glyceraldehyde 3-phosphate dehydrogenase gene (GbGAPDH, L26924) was used as the internal reference gene as described by Cheng et al. (2013). 


\section{Extraction and determination of Terpene lactones}

Ginkgolide A, ginkgolide $B(G B)$, ginkgolide $C(G C)$, and bilobalide $(B B)$ were extracted and their levels were determined using gas chromatography with a wide bore capillary column as Liao (2015) described. The total terpene lactone contents was determined as the sum of the Ginkgolide A, GB, GC, and BB in G. biloba, and sesquiterpene lactone calculated as the contents of BB. All tests were carried out in triplicate, and data represent the means \pm standard errors.

\section{DNA and RNA extraction}

Genomic DNA was extracted from fresh leaves of $G$. biloba using the improved CTAB(Cetyltrimethyl ammonium bromide) method as described by Jiang (2000). Total RNA was isolated from different treatment of Ginkgo leaves using the method of Cheng(2013), followed by incubation with RNase-free DNase I (TaKaRa, Dalian, China) at $37^{\circ} \mathrm{C}$ for $30 \mathrm{~min}$. The quality and concentration of genomic DNA and RNA were determined by agarose gel electrophoresis and spectrophotometer analysis, respectively.

\section{Bioinformatics analysis}

The obtained sequences were analyzed using online bioinformatics tools (http://www. ncbi.nlm.nih.gov). The sequence region was analyzed using the plant cis-acting regulatory element (CARE) database (http://www.dna.affrc.go.jp/PLACE and http:// bioinformatics.psb.ugent.be/ webtools/ plantcare/html/).

\section{RESULTS}

\section{Identification and characteristics of MECT and MECPs specific promoters}

Promoter element analysis showed that Ginkgo MECPs and MECT gene promoter sequences contain a large number of cis-acting elements (Figure 1, Table 2). Therein, the basal promoter element, TATA box, is located $28 \mathrm{bp}$ upstream of the transcription start site of the MECPs gene. The CAAT box is located -53 bp upstream of the transcription start site and the MECT is located between -106 and $-150 \mathrm{bp}$. In addition, there are a further five TATA boxes and seven CAAT boxes in the promoter, indicating that it had strong transcription signals. Table 1 summarizes the different components contained within the promoter fragment, and the results of the preliminary forecast are as follows: GT-1 motif (GRWAAW) (Terzaghi and Cashmore, 1995) contains seven MECPs and MECT, and it is a conserved regulatory site in light-regulated genes; MECPs has three Circadian boxes (CAANNNNATC) (Piechulla et al., 1998), while MECT has none, which is a typical response element to circadian rhythms; GATA box (GATA) (Gilmartin et al., 1990), I-box (GATAA) (Terzaghi and Cashmore 1995) represent the acting elements that are closely related to light response and regulation. As for the acting elements associated with hormone responses, there is one main auxin response element GH3 (CACGCAAT) (Ulmasov et al., 1995), one gibberellin regulatory element GARE-motif (TAACAGA) (Sutoh and Yamauchi, 2003), three WRKY-motifs (TGAC) (Zhang et al., 2004), one ethylene-responsive element ACS (TAAAATAT) (Matarasso et al., 2005), and 15 elements related to cytokinin ARR1 (NGATT) (Ross et al., 2004), one CPBCSPOR (TATTAG) (Fusada et al., 2005), one abscisic acid response element ABRE (ACGTG) (Nakashima 
et al., 2006), and two Dc3 elements (ACACNNG) (Kim et al., 1997). Furthermore, regulatory elements that are associated with stress include MYB and MYC transcription factor binding sites, including five MYB1AT(WAACCA) (Abe et al., 2003), two MYBCORE(CNGTTR) (Urao et al., 1993), one ATMYB2 (CTAACCA) (Abe et al., 1997), one MYCATRD22 (CACATG) (Chinnusamy et al., 2003), and 18 CBF3 (CANNTG) (Rieping and Schöffl, 1992). These regulatory elements are mainly related to environmental stimuli such as dehydration, drought, and cold resistance; MYB and MYC may mediate stress responses by acting as transcription factors. The sequence has been found to contain a high temperature stress response element HSE-motif (CCAAT) (Rieping and Schöffl, 1992) and the cis-acting elements associated with the biological disease As-1 (TTGAC) (Chen and Chen 2002).

In addition to elements related to light, hormones, and adverse stress, the GbMECT promoter sequence also contains the following specific elements: eight root tissue-specific elements rolD (ATATT) (Elmayan and Tepfer 1995), which are located at $-797,-487,-298$, $-221,-214,-181,-160$, and $-137 \mathrm{bp}$; elements related to phenylalanine and lignin metabolism MYBPLANT (MACCWAMC, -685 bp) (Tamagnone et al., 1998); sugar inhibition elements SRE (TTATCC) (Tatematsu et al., 2005) located at $-595 \mathrm{bp}$; oxidative stress and the copper ion response element CuRE (GTAC) (Quinn et al., 2002) located at -414 bp; and an amylase response element amylase-box (TATCCAT, -594 bp) (Hwang et al., 1998) .
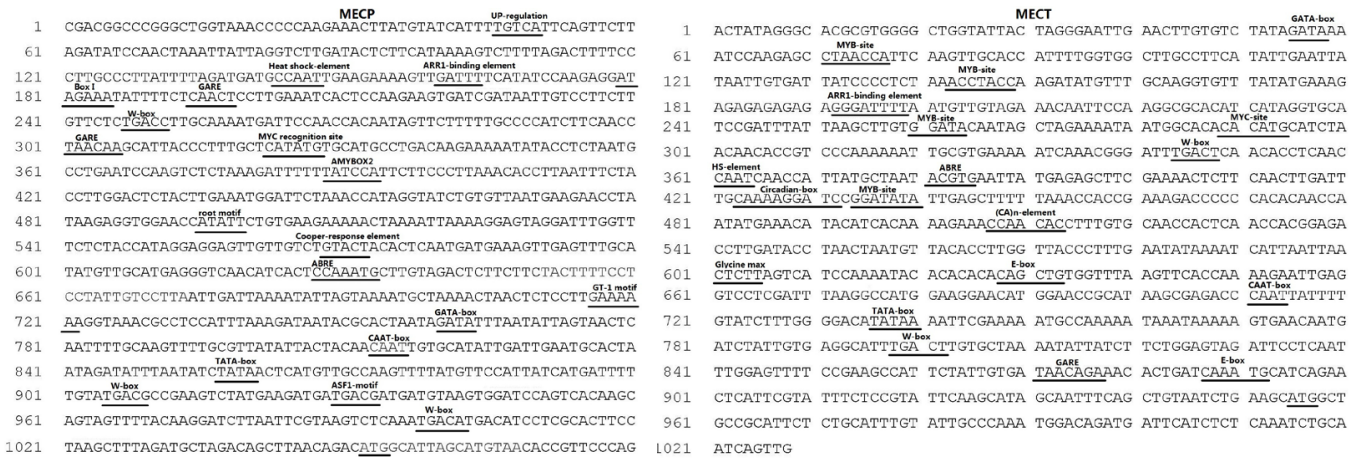

Figure 1. Nucleotide sequence (part) and in silico prediction of TBFs of the GbMECT and MECP promoter region.

\section{Response of MECT and MECPs-specific promoters to wounding, CCC, and UV-B}

To confirm that gene expression was regulated by regulatory elements in the promoter region, we investigated the response to three treatments: WOU, CCC, and UV-B, and employed qRT-PCR to test the influence of these treatments on MECT and MECPs gene transcription (Figure 2). The results show that all injurious treatments led to abnormal expression of the MECT and MECP genes, and were found to significantly induce MECT gene expression. The highest expression of each gene was observed after $12 \mathrm{~h}$, and then decreased slowly. CCC treatment significantly induced MECP gene expression, and its transcriptional level was maximal $12 \mathrm{~h}$ after treatment, after which it declined. CCC treatment had no significant effect on MECT gene expression. MECP was strongly induced under the UV-B treatment, and expression increased rapidly after $4 \mathrm{~h}$, decreased at the $8 \mathrm{~h}$ sampling point, and reached the highest level at $12 \mathrm{~h}$ before a rapid decline thereafter. Conversely, the effect of UV-B on MECT induction was slow, and expression increased slightly at $8 \mathrm{~h}$, and was $\sim 1.2$-fold higher at $12 \mathrm{~h}$, which was maintained thereafter. 
GbMECT and GbMECP gene promoters from G. biloba

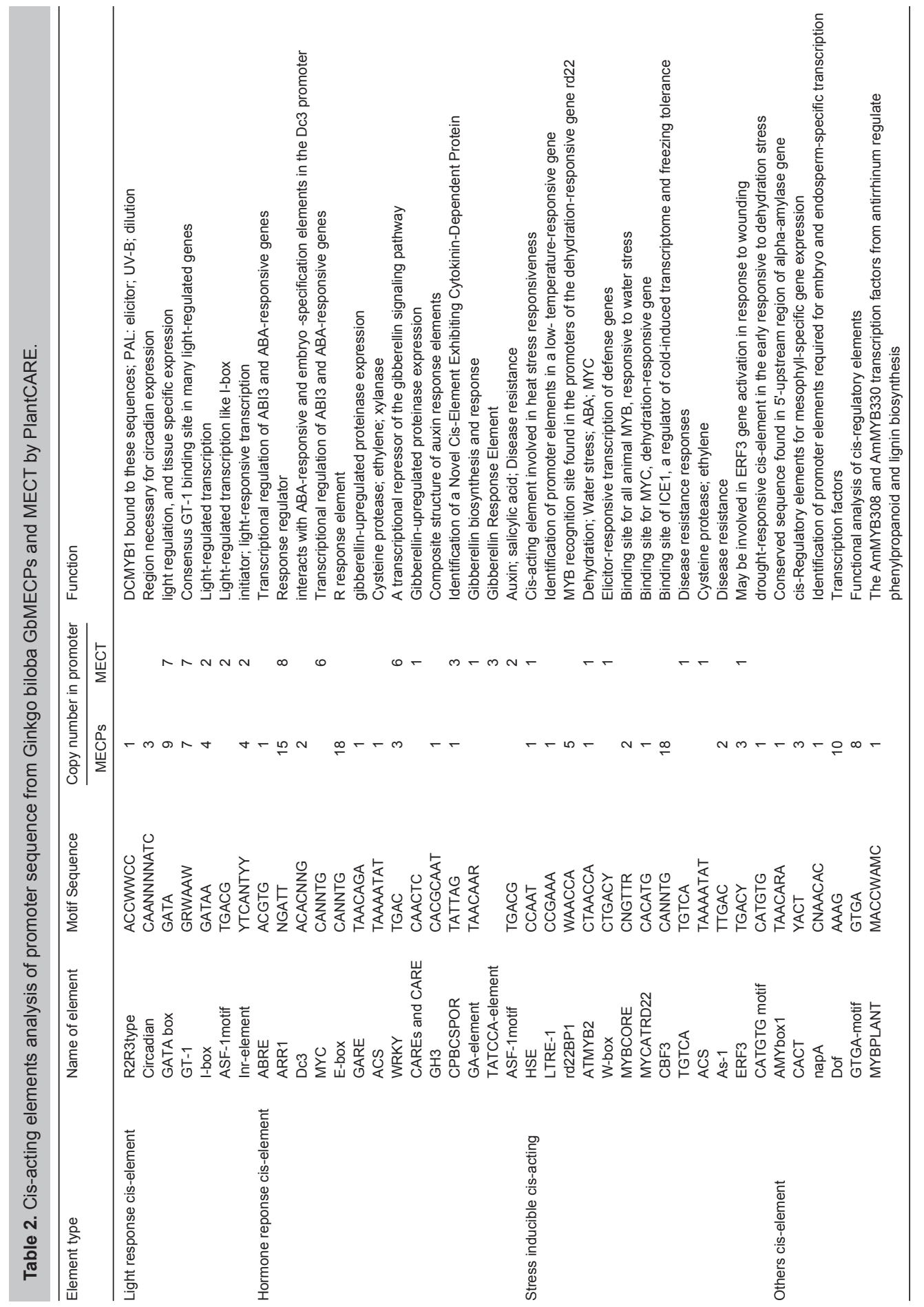




\section{Relationship between the MECT and MECPs genes and levels of terpene lactones}

To study the relationship between terpene content and the expression of two genes of interest, annual changes between the two parameters measured during the growth of Ginkgo leaves were investigated. Grafted Ginkgo leaves were collected at seven sampling points (May12; June 13; July 18; August 13; September 11; October 17; November 14, 2013) for RT-PCR analysis and to measure the terpene lactone content. Figure 3 shows changes in the sesquiterpene content in G. biloba under natural growth conditions. From May to August, the sesquiterpene content in $G$. biloba increased slowly, and quickly rose to the highest annual level from August to October (5.05 $\mathrm{mg} / \mathrm{g}$ ). Figure 4 demonstrates the changing content of total triterpenoids from G. biloba. From May to July, the total triterpenoid content rapidly rose and peaked at $\sim 7.9 \mathrm{mg} / \mathrm{g}$ in August, decreased rapidly to $6.7 \mathrm{mg} / \mathrm{g}$ in September, and rose to the highest annual level of $8.67 \mathrm{mg} / \mathrm{g}$ in October, before decreasing slightly to $7.71 \mathrm{mg} / \mathrm{g}$ in November.

However, the changes in gene expression varied considerably between MECPs and MECT. Expression of MECP declined slightly from May to June and increased afterwards until the highest annual level of 6.47-fold was reached in October, but fell sharply in November to 4.25fold. Expression of MECT increased rapidly from May to July, a 6.7-fold increase, subsequently declining rapidly to the lowest level of 3.48 -fold in November.

A curve-fitting model was implemented between the annual periodic changes and gene expression and total terpenoids. There was a significant quadratic correlation between the level of MECP transcription and the content of terpene lactones in G. biloba, with a correlation coefficient of 0.862 and a correction coefficient of 0.714 (Table 3$)$. The relevant equation is as follows: $Y=(0.999$ $\pm 0.155) X^{2}-(10.372 \pm 1.670) X+(30.443 \pm 4.453)$, where $Y$ is the change in total terpene lactones content and $\mathrm{X}$ is the level of MECP transcription (Table 4 and 5). But there was a weak correlation between MECT and terpene lactones content in leaves. The regression analysis result showed the two genes have no obvious relationship with the sesquiterpenes contents in annual growth year.

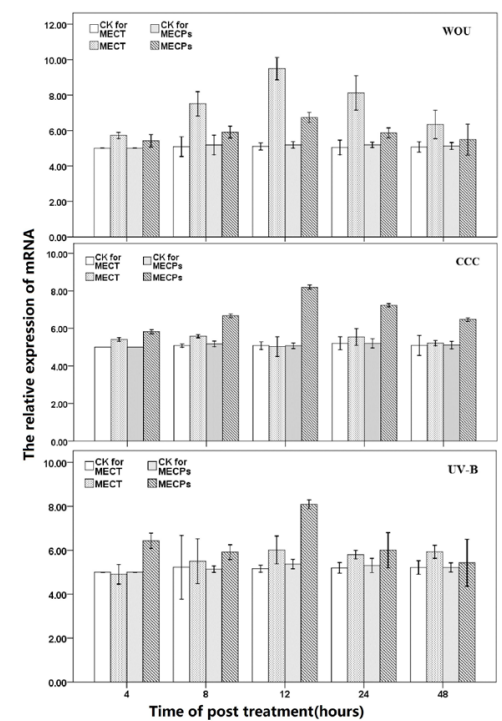

Figure 3. Relationship between the relative expression of MECPS and MECT and the diterpenoid content in Ginkgo leafs by month. 


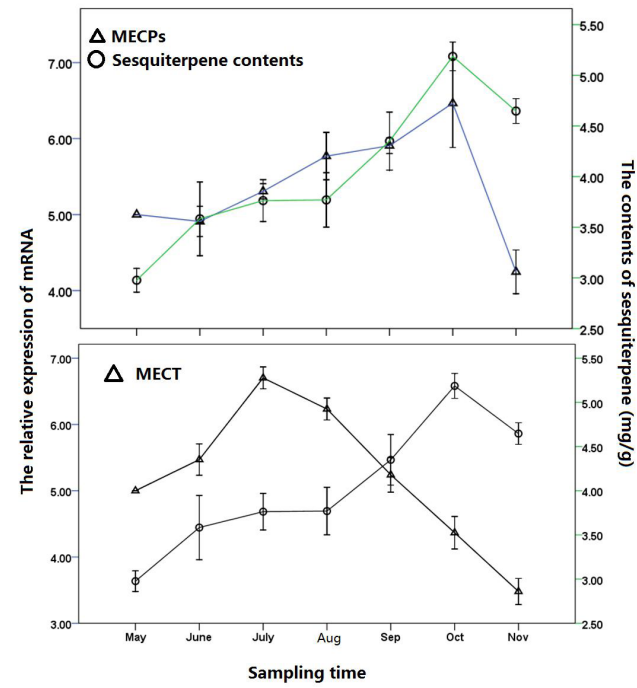

Figure 4. Relationship between the relative expression level of MECPs and MECT and the terpene lactone content in Ginkgo leafs by month.

Table 3. Coefficient change of quadratic model determination.

\begin{tabular}{lccc}
\hline$R$ & $R$ Square & Adjusted R Square & Std. Error of the Estimate \\
\hline 0.862 & 0.742 & 0.714 & 0.377 \\
\hline
\end{tabular}

The independent variable is MECP.

Table 4. Inspection of the quadratic model variance analysis.

\begin{tabular}{lcccr}
\hline & Sum of Squares & Df & Mean Square & F \\
\hline Regression & 7.367 & 2 & 3.683 & 25.915 \\
Residual & 2.558 & 18 & 0.142 & 0.000 \\
Total & 9.925 & 20 & & \\
\hline
\end{tabular}

The independent variable is MECP.

Table 5. Coefficient of the test results of the models between GbMECP and terpene lactones contents.

\begin{tabular}{|c|c|c|c|c|c|}
\hline & \multicolumn{2}{|c|}{ Unstandardized coefficients } & \multirow{2}{*}{$\begin{array}{c}\text { Standardized coefficients } \\
\text { Beta }\end{array}$} & \multirow[t]{2}{*}{$t$} & \multirow[t]{2}{*}{ Sig. } \\
\hline & B & Std. Error & & & \\
\hline MECP & -10.372 & 1.670 & -10.402 & -6.212 & 0.000 \\
\hline $\mathrm{MECP}^{\star \star 2} 2$ & 0.999 & 0.155 & 10.811 & 6.456 & 0.000 \\
\hline (Constant) & 30.443 & 4.453 & & 6.836 & 0.000 \\
\hline
\end{tabular}

\section{DISCUSSION}

Secondary metabolism in plants is affected not only by primary metabolism, but also by the direct regulation of environmental factors (Jacobs and Browner, 2000). Ginkgolides are secondary metabolites in plants, and their level is influenced by integrated conditions including 
illumination, temperature, and soil moisture. Changes in the external environment may also have an impact on the synthesis of endogenous plant hormones such as auxin, gibberellin, ethylene, and ABA (abscisic acid). Some hormones can induce or inhibit the expression and transcription of genes related to ginkgolide synthesis, by affecting the rate or direction of certain precursors in the ginkgolide biosynthesis as well as affecting the ginkgolide content.

The influence of light conditions on biosynthesis and accumulation of ginkgolides has been rarely reported. Leng (2002) explored the effects of light quality and intensity on ginkgolide levels, and found that they are significantly affected by light quality. Whether this is because light quality promotes the expression of genes related to ginkgolide synthesis remains unclear. The ultravioletresponsive element ACCWWCC present in the GbMECPs promoter sequence also exists in the carrot PAL1 promoter sequence, and it has been found that after UV-B irradiation, DCMYB1 can promote PAL1 gene expression by binding to this conserved sequence (Maeda et al., 2005). In addition to the light regulatory conserved element GT-1 (GRWAAW), the Gingko MECPs gene promoter also contains a cis-acting element that is responsive to circadian rhythms, the Circadian box (CAANNNNATC), the presence of which has been confirmed in arabidopsis and other plants (Wan et al., 2010). Hence, expression of MECP induced by UV-B should be related to a regulatory response involving the UV-responsive element ACCWWCC.

Studies have shown that exogenous CCC can significantly induce the expression of four key genes (DXS, DXR, GGPPS, LPS) involved in the biosynthesis pathways of ginkgolides, indicating that CCC may promote the biosynthesis of ginkgolides by inducing the upregulation of key gene expression at the molecular level (Xu et al., 2011). Analysis of expression in ginkgo showed that CCC treatment could also significantly induce the transcriptional upregulation of MECPS. Seasonal analysis of sesquiterpenes in G. biloba revealed that the level of MECPS transcription was significantly correlated with sesquiterpenes and total terpene lactones; therefore, we speculate that MECPs is a key gene involved in ginkgolide biosynthesis.

When tobacco plants are injured exogenously, the WRKY gene quickly binds to W-box elements and induces the expression of related genes (Nishiuchi et al., 2004). GbMECT contains one significant W-box, which is not present in GbMECP; this is consistent with our results showing increased MECT gene expression following wounding. It is therefore presumed that the increase in ginkgolides in response to injury is closely related to the expression of GbMECT. Mild drought leads to the accumulation of ginkgolides in leaves and roots, and the HMGR and MVD genes regulate the metabolism of $B B$ in the MVA pathway, whilst DXS, DXR, MECT, and MECPs regulate total diterpene lactones biosynthesis in the MEP pathway (Zhu, 2010). It is speculated that the environment stimulate the transcription level of MECPs gene to improved the diterpene lactones biosynthesis in MEP pathway. Table 2 shows that GbMECPs and GbMECT contain multiple regulatory elements, including MYB1AT, rd22BP1, ATMYB2, and MYCATRD22, which are stimulated by water shortage. We speculate that MYB and MYC act as transcription factors to mediate adversity stress like drought (Abe et al., 1997). Therefore, these regulatory elements may be involved in the drought stress response of the GbMECPs and GbMECT genes. Functional research, and investigation of seasonal changes in GbMECPs and GbMECT expression and promoter analysis involved in ginkgolide biosynthesis will help us understand how ginkgolide biosynthesis is induced by the environment.

\section{Conflicts of interest}

The authors declare no conflict of interest. 


\section{ACKNOWLEDGMENTS}

Research supported by the National Natural Science Foundation of China \#31400556 and \#31370680, the Natural Science Foundation of Hubei Province (\#2013CFB471), The Open Research Foundation of Economic Forest Germplasm Improvement and Comprehensive Utilization of Resources of Hubei Key Laboratories (\#2013000203).

\section{REFERENCES}

Abe H, Yamaguchi-Shinozaki K, Urao T, Iwasaki T, et al. (1997). Role of Arabidopsis MYC and MYB homologs in drought-and abscisic acid-regulated gene expression. Plant Cell 9: 1859-1868.

Abe $\mathrm{H}$, Urao $\mathrm{T}$, Ito $\mathrm{T}$, Seki M, et al. (2003). Arabidopsis AtMYC2 (bHLH) and AtMYB2 (MYB) function as transcriptional activators in abscisic acid signaling. Plant Cell 15: 63-78.

Bick JA and Lange BM (2003). Metabolic cross talk between cytosolic and plastidial pathways of isoprenoid biosynthesis: unidirectional transport of intermediates across the chloroplast envelope membrane. Arch. Biochem. Biophys. 415: 146-154

Chen $C$ and Chen $Z$ (2002). Potentiation of developmentally regulated plant defense response by AtWRKY18, a pathogeninduced Arabidopsis transcription factor. Plant Physiol. 129: 706-716.

Cheng H, Li LL, Cheng SY, Cao FL, et al. (2013). Molecular cloning and characterization of three genes encoding dihydroflavonol4-reductase from Ginkgo biloba in anthocyanin biosynthetic pathway. PloS One 8: e72017.

Chinnusamy V, Ohta M, Kanrar S, Lee BH, et al. (2003). ICE1: a regulator of cold-induced transcriptome and freezing tolerance in Arabidopsis. Genes Dev. 17: 1043-1054.

Elmayan T and Tepfer M (1995). Evaluation in tobacco of the organ specificity and strength of therolD promoter, domain A of the $35 \mathrm{~S}$ promoter and the 35S2 promoter. Transgenic Res. 4: 388-396.

Fusada N, Masuda T, Kuroda H, Shimada H, et al. (2005). Identification of a novel cis-element exhibiting cytokinin-dependent protein binding in vitro in the 5'-region of NADPH-protochlorophyllide oxidoreductase gene in cucumber. Plant Mol. Biol. 59: 631-645.

Gao S, Lin J, Liu X, Deng Z, et al. (2006). Molecular cloning, characterization and functional analysis of a 2C-methyl-D-erythritol 2, 4-cyclodiphosphate synthase gene from Ginkgo biloba. J. Biochem. Mol. Biol. 39: 502.

Gilmartin PM, Sarokin L, Memelink J and Chua NH (1990). Molecular light switches for plant genes. Plant Cell 2: 369-378.

Hwang YS, Karrer E, Thomas BR, Chen L, et al. (1998). Three cis-elements required for rice a-amylase Amy3D expression during sugar starvation. Plant Mol. Biol. 36: 331-341.

Jacobs BP and Browner WS (2000). Ginkgo biloba: a living fossil. Am. J. Med. 108: 341-342.

Jiang L and Cai LH (2000). A method for extracting DNA of Ginkgo biloba. Plant Physiol. Communicat. 36: 340-341.

Kang MK, Nargis S, Kim SM and Kim SU (2013). Distinct expression patterns of two Ginkgo biloba 1-hydroxy-2-methyl-2-(E)butenyl-4-diphosphate reductase/isopentenyl diphosphate synthase (HDR/IDS) promoters in Arabidopsis model. Plant Physiol. Biochem. 62: 47-53.

Kim SM, Kuzuyama T, Chang YJ, Kwon HJ, et al. (2006). Cloning and functional characterization of 2-C-methyl-D-erythritol 4-phosphate cytidyltransferase (GbMECT) gene from Ginkgo biloba. Phytochemistry 67: 1435-1441.

Kim SY, Chung HJ and Thomas TL (1997). Isolation of a novel class of bZIP transcription factors that interact with ABAresponsive and embryo-specification elements in the Dc3 promoter using a modified yeast one-hybrid system. Plant $\mathrm{J}$. 11: $1237-1251$.

Leng P and Su S (2002). Effects of light intensity and light quality on photosynthesis flavonol glycoside and terpene lactone contents of Ginkgo biloba L. seedlings. J. Plant Res. Environ. 11: 1-4.

Liao Y, Xu F, Huang X, Zhang W, et al. (2015). Promoter analysis and transcriptional profiling of Ginkgo biloba 3-hydroxy-3methylglutaryl coenzyme A reductase gene in abiotic stress responses. Not. Bot. Horti. Agrobo. 17: 146-149.

Lichtenthaler HK (1999). The 1-deoxy-D-xylulose-5-phosphate pathway of isoprenoid biosynthesis in plants. Annu. Rev. Plant Physiol. Mol. Biol. 50: 47-65.

Maeda K, Kimura S, Demura T, Takeda J, et al. (2005). DcMYB1 acts as a transcriptional activator of the carrot phenylalanine ammonia-lyase gene (DcPAL1) in response to elicitor treatment, UV-B irradiation and the dilution effect. Plant Mol. Biol. 59: 739-752.

Matarasso N, Schuster S and Avni A (2005). A novel plant cysteine protease has a dual function as a regulator of 1-aminocyclopropane-1-carboxylic acid synthase gene expression. Plant Cell 17: 1205-1216.

Nakashima K, Fujita Y, Katsura K, Maruyama K, et al. (2006). Transcriptional regulation of ABI3-and ABA-responsive genes including RD29B and RD29A in seeds, germinating embryos, and seedlings of Arabidopsis. Plant Mol. Biol. 60: 51-68. 
Nishiuchi T, Shinshi H and Suzuki K (2004). Rapid and transient activation of transcription of the ERF3 gene by wounding in tobacco leaves possible involvement of NtWRKYs and autorepression. J. Biol. Chem. 279: 55355-55361.

Piechulla B, Merforth N and Rudolph B. (1998). Identification of tomato Lhc promoter regions necessary for circadian expression. Plant Mol. Biol. 38: 655-662.

Quinn JM, Eriksson M, Moseley JL and Merchant S (2002). Oxygen deficiency responsive gene expression in Chlamydomonas reinhardtii through a copper-sensing signal transduction pathway. Plant Physiol. 128: 463-471.

Rieping M and Schöffl F (1992). Synergistic effect of upstream sequences, CCAAT box elements, and HSE sequences for enhanced expression of chimaeric heat shock genes in transgenic tobacco. Mol. Gen. Genet. 231: $226-232$.

Ross EJ, Stone JM, Elowsky CG, Arredondo-Peter R, et al. (2004). Activation of the Oryza sativa non-symbiotic haemoglobin-2 promoter by the cytokinin-regulated transcription factor, ARR1. J. Exp. Bot. 55: 1721-1731.

Smith J and Luo Y (2004). Studies on molecular mechanisms of Ginkgo biloba extract. Appl. Microbiol. Biotechnol. 64: $465-472$.

Sutoh K and Yamauchi D (2003). Two cis- acting elements necessary and sufficient for gibberellin-upregulated proteinase expression in rice seeds. Plant J. 34: 635-645.

Tamagnone L, Merida A, Parr A, Mackay S, et al. (1998). The AmMYB308 and AmMYB330 transcription factors from Antirrhinum regulate phenylpropanoid and lignin biosynthesis in transgenic tobacco. Plant Cell 10: 135-154.

Tatematsu K, Ward S, Leyser O, Kamiya Y, et al. (2005). Identification of cis-elements that regulate gene expression during initiation of axillary bud outgrowth in Arabidopsis. Plant Physiol. 138: 757-766.

Terzaghi WB and Cashmore AR. (1995). Light-regulated transcription. Annu. Rev. Plant Physiol. Plant Mol. Biol. 46: 445-474.

Ulmasov T, Liu ZB, Hagen G and Guilfoyle TJ (1995). Composite structure of auxin response elements. Plant Cell 7: $1611-1623$.

Urao T, Yamaguchi-Shinozaki K, Urao S and Shinozaki K (1993). An Arabidopsis myb homolog is induced by dehydration stress and its gene product binds to the conserved MYB recognition sequence. Plant Cell 5: 1529-1539.

van Beek TA and Montoro P (2009). Chemical analysis and quality control of Ginkgo biloba leaves, extracts, and phytopharmaceuticals. J. Chromatogr. A 1216: 2002-2032.

Veau B, Courtois M, Oudin A, Chénieux JC, et al. (2000). Cloning and expression of cDNAs encoding two enzymes of the MEP pathway in Catharanthus roseus. Biochim. Biophys. Acta 1517: 159-163.

Wan X, Mo A, Pan J and Liang C (2010). Molecular cloning and GUS aided activity analysis of promoter region sequence of AtNCED2 gene from Arabidopsis thaliana L. Biotechnology 20: 18-23.

Xu F, Zhang W, Sun N, Li L, et al. (2011). Effects of chlorocholine chloride on photosynthesis metabolism and terpene trilactones biosynthesis in the leaf of Ginkgo biloba. Acta Hortic. 38: 2253-2260.

Zhang ZL, Xie Z, Zou X, Casaretto J, et al. (2004). A rice WRKY gene encodes a transcriptional repressor of the gibberellin signaling pathway in aleurone cells. Plant Physiol. 134: 1500-1513.

Zhu CC (2010). Effects of environmental factors on the growth and principal medicinal compositions of Ginkgo biloba seedlings. Doctoral thesis, Nanjing Forestry University, Nanjing. 